\title{
Effects of Halo on the AGS Injection from 1.2Gev Linac*
}

\author{
W.T. Weng**, J. Beebe-Wang, D. Raparia, A. G. Ruggiero, N. Tsoupas \\ Brookhaven National Laboratory, Upton, NY 11766
}

\begin{abstract}
BNL is conducting a design study of a $1.0 \mathrm{MW}$ super neutrino beam facility. It requires 230 turns charge exchange injection from a $1.2 \mathrm{GeV}$ superconducting linac with $28 \mathrm{~mA}$ current for $0.72 \mathrm{msec}$. This report studies the impact of halo distribution of the linac beam on the efficiency of injection and the final beam distribution in the AGS as functions of the injection orbit bump and the foil thickness. Another important consideration is the residual radiation generated on the accelerator components near the injection area. If necessary, radiation hardened components and local shielding have to be provided.
\end{abstract}

\section{INTRODUCTION}

We have examined [1] possible upgrades to the AGS complex that would meet the requirements of the proton beam for a 1.0 MW neutrino superbeam facility. We are proposing to build a superconducting upgrade to the existing $200 \mathrm{MeV}$ linac to an energy of $1.2 \mathrm{GeV}$ for direct $\mathrm{H}^{-}$injection into the AGS.

The requirements of the proton beam for the super neutrino beam are summarized in Table 1 and a layout of upgraded AGS is shown in Figure 1. Since the present number of protons per fill is already close to the required number, the upgrade focuses on increasing the repetition rate and reducing beam losses (to avoid excessive shielding requirements and to maintain activation of the machine components at workable level). It is also important to preserve all the present capabilities of the AGS, in particular its role as injector to RHIC.

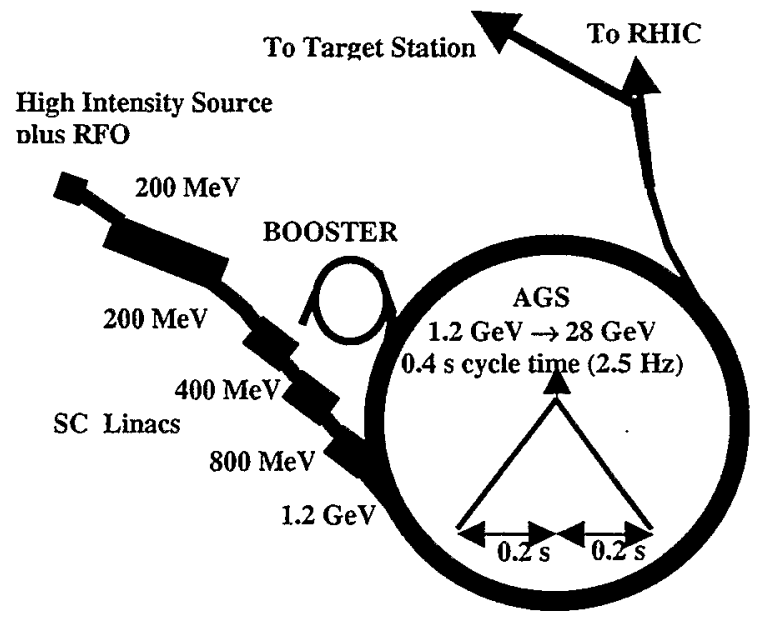

FIGURE 1. Schematic diagram of the accelerators for the "neutrino production".

\footnotetext{
* Work performed under the auspices of the US Department of Energy

** E-mail weng@bnl.gov
}

TABLE 1. AGS Proton Driver Parameters.

\begin{tabular}{ll}
\hline Total beam power & $1 \mathrm{MW}$ \\
Beam energy & $28 \mathrm{GeV}$ \\
Average beam current & $42 \mu \mathrm{A}$ \\
Cycle time & $400 \mathrm{msec}$ \\
Number of protons per fill & $0.9 \times 10^{14}$ \\
Number of bunches per fill & 24 \\
Protons per bunch & $0.4 \times 10^{13}$ \\
Injection turns & 230 \\
Repetition rate & $2.5 \mathrm{~Hz}$ \\
Pulse length & $0.72 \mathrm{msec}$ \\
Chopping rate & 0.75 \\
Linac average/peak current & $20 / 30 \mathrm{~mA}$ \\
\hline
\end{tabular}

Present injection into the $A G S$ requires the accumulation of four Booster loads in the AGS which takes about $0.6 \mathrm{sec}$, and is therefore not suited for high average beam power operation. To minimize the injection time to about $1 \mathrm{msec}$, a $1.2 \mathrm{GeV}$ linac will be used instead. The injection Linac consists of the existing warm linac of 200 $\mathrm{MeV}$ and a new superconducting linac of $1.0 \mathrm{GeV}$. The multi-turn injection from a source of $28 \mathrm{~mA}$ and $720 \mu$ sec pulse width is sufficient to accumulate $0.9 \times 10^{14}$ particle per puise in the AGS. The minimum ramp time of the AGS to full energy is presently $0.5 \mathrm{sec}$. This must be reduced down to 0.2 sec to reach the required repetition rate of $2.5 \mathrm{~Hz}$ to deliver the required $1 \mathrm{MW}$ beam to the garget.

\section{SUPERCONDUCTING LINAC (SCL)}

The superconducting linacs accelerate the proton beam from $200 \mathrm{MeV}$ to $1.2 \mathrm{GeV}$. The presented configuration follows a design described in detail in [2]. All three linacs are built up from a sequence of similar periods. The major parameters of the three sections of the SCL are given in Table 2. The low energy section operates at $805 \mathrm{MHz}$ and accelerates proton from 200 to $400 \mathrm{MeV}$. The two sections, accelerating to $800 \mathrm{MeV}$ and $1.2 \mathrm{GeV}$, operate at 
$1.61 \mathrm{GHz}$. A higher frequency is desirable for obtaining a larger accelerating gradient with a more compact structure and reduced cost. The SCL will be operated at $2{ }^{\circ} \mathrm{K}$ for reaching the desired gradients.

TABLE 2. General parameters of the SCL.

\begin{tabular}{lccc}
\hline Linac section & LE & ME & HE \\
\hline Average beam power, $\mathrm{kW}$ & 7.14 & 14 & 14 \\
Average beam current, $\mu \mathrm{A}$ & 35.7 & 35.7 & 35.7 \\
Initial kinetic energy, MeV & 200 & 400 & 800 \\
Final kinetic energy, MeV & 400 & 800 & 120 \\
Cell reference $\beta_{0}$ & 0.615 & 0.755 & 0.887 \\
Frequency, MHz & 805 & 1610 & 1610 \\
Cells/cavity & 8 & 8 & 8 \\
Cavities/cryo-module & 4 & 4 & 4 \\
Cavity internal diameter, cm & 10 & 5 & 5 \\
Total length, m & 37.82 & 41.4 & 38.32 \\
Accelerating gradient, $\mathrm{MeV} / \mathrm{m}$ & 10.8 & 23.5 & 23.4 \\
Cavities/Klystron & 1 & 1 & 1 \\
Norm. rms emittance, $\pi \mathrm{mm}$ mrad & 2 & 2 & 2 \\
Ims bunch area, $\pi^{\circ} \mathrm{MeV}(805 \mathrm{MHz})$ & 0.5 & 0.5 & 0.5 \\
\hline
\end{tabular}

\section{H BEAM INJECTION INTO AGS}

The $\mathrm{H}^{-}$injection region has been chosen to be the location of the B20 straight section of the AGS. A schematic diagram of the injection region is shown in Figure 2. In this diagram the following components of the injection region are shown: (a) three of the main magnets of the AGS; (b) the stripping foil; (c) the closed beam orbits; (d) The trajectory of the injected $\mathrm{H}^{-}$beam; (e) the trajectories of the $\mathrm{H}^{-}$beam which is not stripped by the stripping foil; $(f)$ the trajectory of the partially stripped $\mathrm{H}^{-}$beam $\left(\mathrm{H}^{0}\right)$ and (g) the trajectories of the electrons emanating from the stripping foil.

All secondary particles must be collected downstream of the stripping foil into various "beamdumps". In order to make the circulating proton beam collinear with the $\mathrm{H}^{-}$injected beam at the injection point, the circulating proton beam is "locally bumped" by using two horizontal "bumpmagnets" [3].

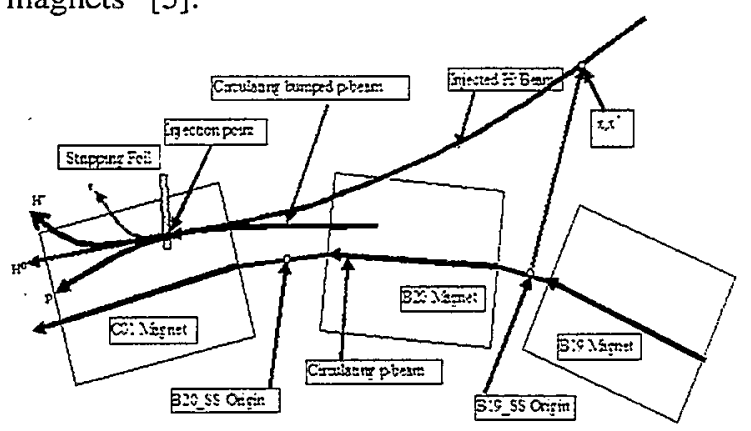

FIGURE 2. Schematic diagram of the injection region.

\section{HALO/TAIL GENERATION VS. LINAC EMITTANCE}

For high intensity proton accelerators, such as the upgraded AGS, there are very stringent limitations on uncontrolled beam losses. In this paper, we present the estimate of emittance growth and uncontrolled beam losses as function of linac emittance by computer simulations.

All of the physical quantities used in the simulations (Table 1 and 3 ) are chosen according $t)$ the design specifications [1]. Correlated Painting |4| is chosen for injection into AGS, considering the available aperture at injection and beam halo/tail control. A significant effort has been made $(0)$ optimize injection painting $14 \mathrm{~J}$. The optimized injection bump collapses as an exponential function of time with a time-constant of $0.1 \mathrm{msec}$. The initial foil-hit by each incident $\mathrm{H}^{-}$is counted as thrice to include the effects of two stripped electrons. The average foil thickness is assumed to be $300 \mu \mathrm{g} / \mathrm{cm}^{2}$. In order to separate the effects of linac emittance from the other issues, the effects of space charge and magnet errors are not included in this study.

TABLE 3 Simulation parameters.

\begin{tabular}{ll}
\hline Horizontal beta at the injection & $28.0 \mathrm{~m}$ \\
Vertical beta at the injection & $8.0 \mathrm{~m}$ \\
Horizontal emittance of injected beam & $2 \pi \mathrm{mm}-\mathrm{mrad}$ \\
Vertical emittance of injected beam & $2 \pi \mathrm{mm}-\mathrm{mrad}$ \\
Horizontal beam size at injection, $\sigma_{\mathrm{x}}$ & $5.2293 \mathrm{~mm}$ \\
Vertical beam size at injection, $\sigma_{\mathrm{y}}$ & $2.7952 \mathrm{~mm}$ \\
Horizontal Foil size $\left(2.5 \sigma_{\mathrm{x}}\right)$ & $13.0731 \mathrm{~mm}$ \\
Vertical foil size $\left(2.5 \sigma_{\mathrm{v}}\right)$ & $6.9878 \mathrm{~mm}$ \\
\hline
\end{tabular}

A direct effect of linac beam emittance is the halo/tail generation in the circulating beam. Figure 3 shows the estimated halo/tail generation in the beam [4] as a function of normalized RMS emittance of linac beam. Here, the Halo/tail generation is defined as the ratio of number of particles with emittance larger than the designed acceptance of $49 \pi \mathrm{mm}$-mrad to the total number of particles in the circulating beam.

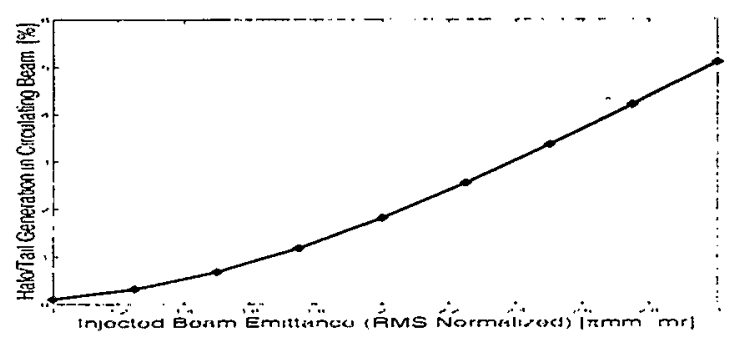

FIGURE 3 The estimated halo/tail generation in the circulating beam as functions of normalized RMS emittance of injected beam. 


\section{INJECTION EFFICIENCY VS. LINAC EMMITANCE}

Injection efficiency is associated with two kinds of beam losses at the injection stripping foil: (1) controlled losses which can be directed to the injection beam dumps located down stream of the injection foil; and (2) uncontrolled losses which are scattered in all directions and generate radiation in the injection area [5].

As a consequence of particle traversal in the stripping foil, there are beam losses associated to: (1) nuclear scattering, (2) energy straggling, and (3) multiple scattering [5].

\section{Nuclear Scattering}

The beam loss due to nuclear scattering in the foil is a function of the foil traversal rate, foil thickness and Linac beam emittance. Figure 4(a) shows the fractional nuclear scattering losses as a function of the normalized RMS Linac beam emittance. If all the losses are located in the injection straight section $(\sim 10 \mathrm{~m})$, the resulting estimated radiation level, at 1 foot from the beam line after a 100 day run followed by 4 hours of shutdown, is shown in Figure 4(b) as a function of normalized RMS emittance of injected beam.
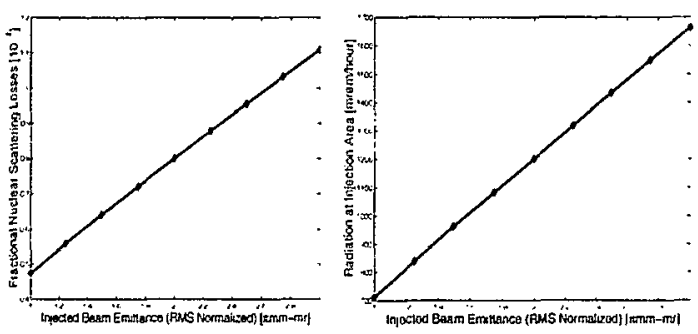

FIGURE 4 (a) Fractional beam losses at injection area and (b) radiation level due to nuclear scattering in the stripping foil as functions of normalized RMS emittance of injected bearn.

\section{Energy Straggling}

As particles traverse through the injection foil, a fraction of their energies are lost and deposited in the foil. Some of the circulating protons, which lie in the tail of Landau distribution, will go through large synchrotron oscillations and may end up in the beam gap. These protons will be lost at the time of extraction or at a dispersive location in the ring. This beam loss is proportional to foil hitting rate, which is associated to the emittance of injected beam when the foil size of $5 \sigma_{\text {inj }}$ is kept. Figure $5(\mathrm{a})$ shows the estimated fractional beam loss in the gap due to energy straggling as functions of normalized RMS emittance of injected beam.

\section{Multiple Scattering}

The major effect of particle multiple scattering in the foil is to increase the transverse beam emittance. Figure $5(\mathrm{~b})$ shows the estimated transverse emittance growth due to multiple scattering as functions of normalized RMS emittance of injected beam.


FIGURE 5 (a) The estimated fractional beam loss in the gap due to energy straggling; (b) the estimated transverse emittance growth due to multiple scattering as functions of normalized RMS emittance of injected beam.

\section{MAXIMUM FOIL TEMPERATURE VS. LINAC EMMITANCE}

The foil temperature distribution is governed by the current density distribution on the foil during injection. Different painting schemes [4] produce not only different final particle distributions, but also generate different foil traversal patterns and therefore different current density distributions in the foil. In this study the current density distributions are simulated through turn-by-turn beam tracking during the correlated painting with the optimized injection bump. The initial foil-hit by each incident $\mathrm{H}^{-}$is counted as thrice to include the effects of two stripped electrons. The foil temperature distributions are then converted from the current density distributions through the relationship presented in Figure 6 . This is calculated from the model described in reference [6] with a foil thickness of $300 \mu \mathrm{g} / \mathrm{cm}^{2}$. The model includes:

1 the radiation heat transfer between the carbon foil and the stainless steel beam pipe,

2 the heat conduction through the foil to its base.

3 a natural convection condition on the outer surface of the beam pipe, and

4 a Gaussian distribution of injected beam.

Figure 7 shows the maximum foil temperature distribution with the injected beam of $2 \pi \mathrm{mm}$-mrad normalized RMS emittance of injected beam.

The maximum foil temperature is a function of the linac beam emittance. For a given foil thickness, the maximum foil temperature decreases while the 
linac beam emittance increases. However, with a foil size of $5 \sigma_{\mathrm{inj}}$, the foil-hitting rate also increases with $\sigma_{\text {inj. }}$. It will induce more uncontrolled beam loss.

The foil lifetime tests at BNL Linac has indicated that the maximum single foil lifetime is $\sim 78$ hours and decrease sharply when the foil temperature exceeds $2500^{\circ} \mathrm{K}$ [7]. In general, the injection foil temperature of upgraded AGS can be kept under $2500^{\circ} \mathrm{K}$. Therefore, upgraded AGS injection foil will have a long lifetime.

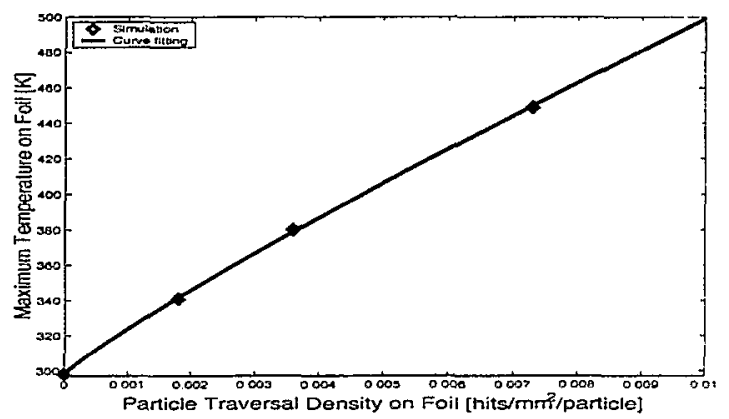

FIGURE 6 Maximum temperature on foil vs. beam current density (blue points) deduced from the model [6] and the curve fit (red line) used in foil temperature distribution studies.

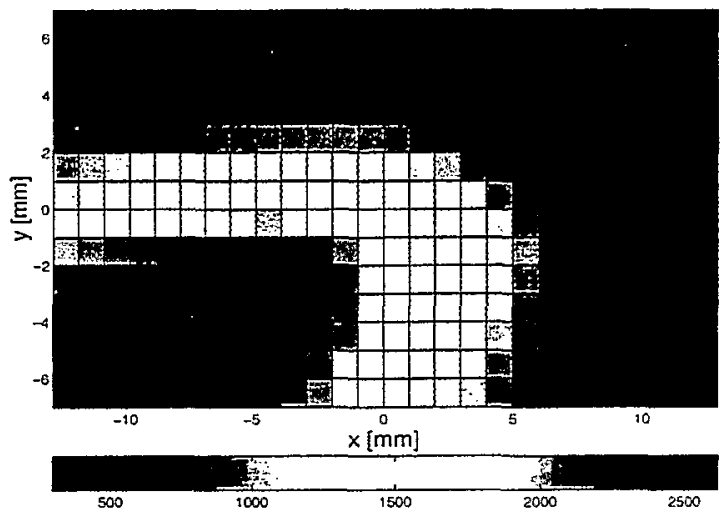

FIGURE 7 Simulation result of the foil temperature distribution. The entire bottom portion of $\mathrm{H}^{-}$injection foil is shown with horizontal coordinate $x$ and vertical coordinate $y$.

\section{CONCLUSIONS AND DISCUSSIONS}

From the results shown above, it is clear that the correlated painting with the optimized injection bump, collapsing as an exponential function of time with a time-constant of $0.1 \mathrm{msec}$, gives the best final proton beam distribution in the upgraded AGS. The preferred linac emittance is about $1.5 \pi \mathrm{mm}$-mrad for acceptable injection losses.

Two methods have been considered to improve the $\mathrm{H}^{-}$injection from linac. One is to bring the RFQ immediately adjacent to the output of the ion source to reduce the emittance growth. In our estimate, this can reduce the linac output emittance from $3 \pi \mathrm{mm}$ mrad to about $1.5 \pi \mathrm{mm}$-mrad. Well-designed low level feedback system has to be provided to prevent further emittance growth in the high-energy section of the linac.

Another possible improvement is to introduce a second harmonics cavity for the AGS during injection time. It can effectively reduce the space charge tune shift by $30 \%$. This will either reduce resonance losses for same intensity, or allow for $30 \%$ more intensity to be accepted by the AGS.

\section{ACKNOWLEDGMENTS}

The authors would like to acknowledge the contributions by the members of the $C-A D$ accelerator physics group. The assistances of C. J. Liaw in the foil temperature simulation and foil lifetime experiments are also greatly appreciated.

\section{REFERENCES}

1. Diwan, M, et al., "AGS Super Neutrino Beam Facility Accelerator and Target System Design", BNL-712282003-IR. April 15. 2003.

2. Ruggiero, A. G., "AGS Upgrade to $1 \mathrm{MW}$ with a SuperConducting Linac Injector" in Proceedings of PAC2003 (2003).

3. Tsoupas,N.,et al."Injection Acceleration and Extraction of High Intensity Proton Beam For The Neutrino Facility Prodject at BNL", in Proceedings of PAC2003.

4. Beebe-Wang. J., et al.. "Beam Properties in the SNS Accumulator Ring due to Transverse Phase Space Painting", Proceedings of the EPAC 2000. Vienna, Austria. 26-30 June 2000, p.1465-1467.

5. Beebe-Wang, J., et al., "Injection Carbon Stripping Foil Issues in the SNS Accumulator Ring". Proceedings of the PAC2001. Vienna, Austria, 26-30 June 2000. p. $1508-1510$.

6. Liaw, C. J., et al. "Calculation of Maximum Temperature on the Carbon Stripping Foil of the Spallation Neutron Source". Proceedings of the PAC99. New York. USA, March 27-April 2, 1999.

7. Liaw, C. J.. el al.. "Life Time of Carbon Stripping Foils For the Spallation Neutron Source" PAC2001. Vienna. Austria. 26-30 June 2000. 